grade serous carcinoma (HGSC) in patients with a BRCA1/2 pathogenic variant (PV), but results in significant short and long-term health risks. New interventions, such as risk-reducing salpingectomy with delayed oophorectomy are promising. In this alternative approach, the detection of serous tubal intraepithelial carcinoma (STIC) as precursor to HGSC, has become more important. The detection of STIC indicates an increased risk for HGSC and would prompt for an immediate oophorectomy. Unfortunately, reproducibility of STIC diagnosis is only moderate, even among experienced gynecological pathologists. The aim of this pilot study is to develop and validate an AI algorithm for automated detection of potential STIC lesions in scanned H\&E slides, to aid the pathologist in diagnosing STIC

Methodology We collected and digitalised 60 cases of STIC and 65 control cases. STIC diagnosis was confirmed using p53 and Ki-67 immunohistochemical stainings (IHC). The dataset was split into 50 cases for training, five for validation and five for testing. We developed a Convolutional Neural Network and compared two appraoches: directly detecting STIC (one-step) or first detecting all epithelium and subsequently detecting STIC within epithelial regions (two-step). Additionaly, we evaluated whether we could improve the network by enriching the training data with hard negative examples.

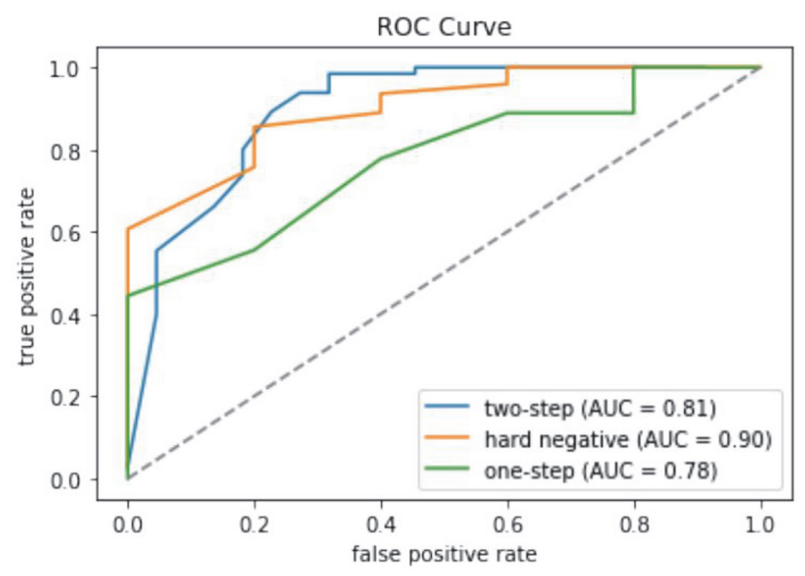

Abstract 1009 Figure 1

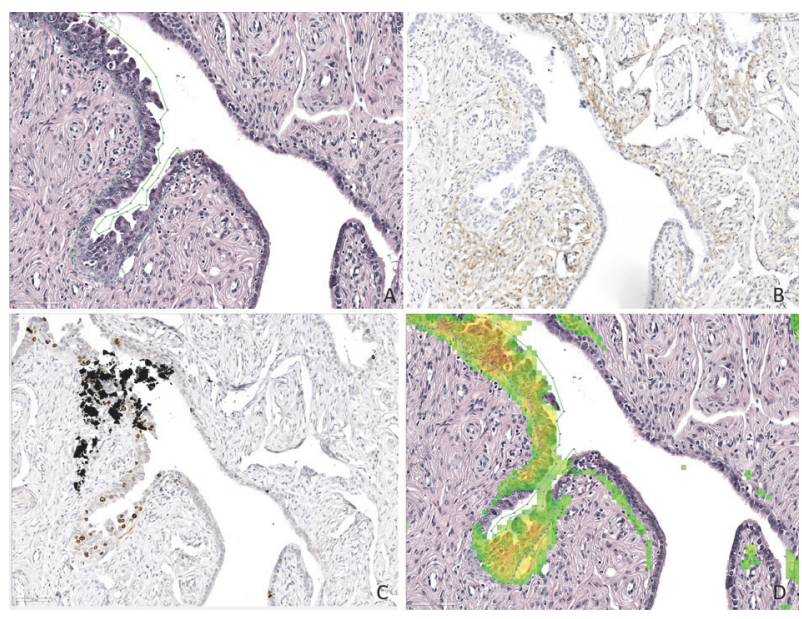

Abstract 1009 Figure 2
Result(s)* We found that the optimal configuration for detection of STIC was the two-step approach, with training set enrichment by hard negatives. This network reached an area under the receiver operating curve of 0.90 (figure 1). Visual inspection of cases in the test set showed concordance between the model output, p53 and Ki67 IHC, and pathologists' annotations (see example model output on H\&E stained tissue in figure 2 .

Conclusion* We present a convolutional neural network that can succesfully detect STIC lesions in whole slide images. AI has the potential to aid the pathologist in the detection of STIC and assist in producing more accurate and consistent diagnosis. Additional performance and robustness is expected to be achieved by expansion of the dataset.

\section{UNUSUALLY BREAST METASTASES}

${ }^{1} S$ Sakhri, ${ }^{2} \mathrm{~K}$ Tlili, ${ }^{3} \mathrm{~S}$ Kammoun, ${ }^{4} \mathrm{M}$ Bouhani*, ${ }^{5} \mathrm{Y}$ Houcine, ${ }^{3} \mathrm{~K}$ Hamza, ${ }^{2} \mathrm{H}$ Bouaziz, ${ }^{6} \mathrm{~A}$ Ghoucha, ${ }^{6} \mathrm{I}$ Bettaib, ${ }^{6} \mathrm{M}$ Idriss. 'Institut Salah Azaiz, Surgical Departement, Tunis, Tunisia; ${ }^{2}$ Azaiez Institute of Oncology, Department of Pathology, Tunisia; ${ }^{3}$ Salah Azaiez Institute, anatomopathology, Tunis, Tunisia; ${ }^{4}$ Salah Azaiez Institute, surgical departement, Tunsia, Tunisia; ${ }^{5}$ nstitut Salah Azaiz, anatomopathology, Tunis, Tunisia; ${ }^{6}$ nstitute Salah Azaïz, anatomopathology, tunsia, Tunisia

\subsection{6/ijgc-2021-ESGO.534}

Introduction/Background* The breast is the most common site of primary malignancies in adult women, but is an uncommon site for metastasis from extramammary malignancies. Breast metastases from extramammary malignancies have both hematogenous and lymphatic routes. We report 10 cases of breast metastases in our pathology lab between 2014 and 2020 .

Methodology We have identified all the cases of breast metastases diagnosed between 2014 and 2020.

Result(s)* We found 10 cases of breast metastases from extra mammary malignancies. All patients was female with a middle age of 42 years. The primary malignancies was a melanoma in 3 cases, an ovarian carcinoma also in 3 cases, a fibro sarcoma, a rhabdomyosarcoma and a nasopharyngeal carcinoma in 1 case each. The diagnosis was based on morphological features but also on immunohistochemistry especially with the ovarian carcinoma which exhibit some similar features to a primary breast carcinoma.

Conclusion* Breast metastases from extramammary malignancies show variable histological features. Therefore, the possibility of metastatic lesion should be considered in evaluating breast lesions in patients with primary malignancy in other organs. Awareness of typical and atypical imaging features of such lesions may be helpful to diagnose metastatic lesions in the breast.

\section{ATYPICAL (C3) AND SUSPICIOUS (C4)CATEGORIES INBREAST FINE NEEDLE ASPIRATION}

${ }^{1} S$ Sakhri, ${ }^{2} \mathrm{~K}$ Hamza, ${ }^{2} \mathrm{Y}$ Houcine, ${ }^{2} \mathrm{~S}$ Kammoun, ${ }^{2} \mathrm{~K}$ Tlili, ${ }^{3} \mathrm{M}$ Bouheni ${ }^{*},{ }^{2} \mathrm{~A}$ Goucha, ${ }^{2}$ I Bettaieb, ${ }^{2} \mathrm{M}$ Driss. ${ }^{1}$ Institute Salah Azaï, surgical departement, Tunis, Tunisia; ${ }^{2}$ Institut Salah Azaiz, anatomopathology, Tunis, Tunisia; ${ }^{3}$ Institut Salah Azaiz, surgical departement, Tunis, Tunisia

\subsection{6/ijgc-2021-ESGO.535}

Introduction/Background* The presentstudywascarried out over a 1-year period (January 2017-December 2017) and conductedat the cytology unit of PathologyDepartment (Salah Azaiez 